\title{
Via de Parto em Gestaçōes Sucessivas em Adolescentes: Estudo de 714 Casos
}

Route of Delivery in Successive Gestations in Adolescents: Study of 714 Cases

Vamberto Oliveira de Azevedo Maia, Alessandra Costa de Azevedo Maia, Fábio Lima Queiroga, Vamberto Oliveira de Azevedo Maia Filho, Aline Brandão de Araújo, Luís André Marinho Lippo, Rivaldo Mendes de Albuquerque

\begin{abstract}
RESUMO
Objetivo: analisar a associação da via de partos consecutivos de 714 gestantes adolescentes, que pariram na Maternidade do Hospital das Clínicas da Universidade Federal de Pernambuco, no periodo de janeiro a dezembro de 2001.

Pacientes e Métodos: em estudo prospectivo, analitico, transversal, tipo incidência, foi analisada a sucessão das vias de parto da primeira até a quarta gestação de 714 parturientes adolescentes, com idade variando entre 13 e 19 anos (média 17,2士1,5 anos). Identificandose, no livro da sala de parto, a cada dia, as adolescentes que pariram, após ter obtido o consentimento livre e esclarecido, cada uma respondeu a um questionário estruturado, com 65 perguntas diretas e respostas fechadas, pré-codificadas, entre as quais estavam vias de parto e o número de gestações.

Resultados: observou-se que $527(73,8 \%)$ adolescentes tiveram uma única parturição, 149 (20,9\%) duas parturições, 35 (4,9\%) três partos e $3(0,4 \%)$ quatro partos. Dentre os 273 partos cesáreos, 207 (75,8\%) ocorreram em primiparas, 65 (23,8\%) em paucíparas e $1(0,4 \%)$ em multípara. Houve associação estatisticamente significante entre via de parto da primeira e da segunda parturições; em 83 (55,7\%) gestantes adolescentes quanto à via transvaginal e em 41 (27,5\%), para a via cesárea. Coincidiram também as vias de parto de segunda e terceira parturições, em 23 (65,7\%) gestantes adolescentes no parto transvaginal e em outras 10 $(28,6 \%)$ na cesárea.

Conclusão: identificou-se tendência da coincidência das vias de parto em gestações sucessivas em parturientes adolescentes, até a terceira parturição
\end{abstract}

PALAVRAS-CHAVE: Gravidez normal. Adolescência. Cesariana. Parto normal.

\section{Introdução}

A gravidez na adolescência é tida, mundialmente, como problema relevante de saúde pública, tanto nos países desenvolvidos como naqueles considerados em vias de desenvolvimento, justificando a grande quantidade de estudos publicados na literatura internacional ${ }^{1}$.

Hospital das Clínicas da Universidade Federal de Pernambuco

Correspondência:

Vamberto Oliveira de Azevedo Maia Filho

Av. Rosa e Silva, 707, apto. 2102

52020-220 - Recife - PE

Telefone: (81) 3241-3257 - Fax : (81) 3427-0032

e-mail: vamberto@smartsat.com.br
Têm sido abordados aspectos biológicos ${ }^{2,3}$, psicossociais $^{4,5}$ e demográficos ${ }^{6-10}$ da gravidez na adolescência, buscando interpretar as conseqüências reais nessa etapa do desenvolvimento, na qual estruturam-se as bases psíquicas e afetivas ${ }^{11}$ que determinarão a qualidade de vida na fase adulta. Silva e Nogueira ${ }^{1}$ ressaltam ainda que a maioria dos trabalhos se detém na análise do comportamento de adolescentes primigestas, deixando de ponderar a eventual situação de multiparidade.

Torres Ramírez et al. ${ }^{11}$ afirmam que os dados referentes à freqüência de partos vaginais e cesáreos entre adolescentes são limitados e contraditórios, já que alguns estudos referem ser a cesárea mais praticada em adolescentes que em adultas, devido à imaturidade biológica das ado- 
lescentes, assim como a um controle pré-natal menor, ao passo que outras pesquisas mostram freqüências idênticas dessa via de parto para adolescentes e adultas, atribuindo essa contradição ao fato de todos os estudos compararem populações em geral, com e sem doenças sistêmicas e com ou sem antecedentes obstétricos.

$\mathrm{Na}$ literatura consultada sobre o tema gestação na adolescência, dentre 118 trabalhos nacionais e internacionais, seis abordam multigestação, mas apenas Silva e Nogueira ${ }^{1}$ relacionam via de parto e número de gestações, sem detalhar esta variável a cada gestação, o que motivou o presente trabalho.

Dessa forma, pareceu ser de interesse, para melhor entendimento do problema gestação na adolescência, considerar adolescentes multigrávidas, já que a casuística disponível para o presente estudo é maior que a de Silva e Nogueira $^{1}$, o que poderá subsidiar dados consistentes para planejamento do atendimento obstétrico a essas pacientes.

Objetivou-se analisar a associação da via de partos de 714 gestantes adolescentes, atendidas na Maternidade do Hospital das Clínicas da Universidade Federal de Pernambuco, no período de janeiro a dezembro de 2001.

\section{Pacientes e Métodos}

Por meio de estudo prospectivo, analítico, transversal, tipo incidência, analisou-se a sucessão das vias de parto da primeira até a quarta gestação de 714 parturientes adolescentes atendidas na Maternidade do Hospital das Clínicas da Universidade Federal de Pernambuco, no período de janeiro a dezembro de 2001. As pacientes apresentavam idades variando entre 13 e 19 anos (média $17,2 \pm 1,5$ anos).

A cada dia, por meio da pesquisa do livro da sala de parto, foram identificadas as parturientes adolescentes e seu respectivo local de internação. Na enfermaria, feita a identificação da gestante, foram explicados, a ela e ao seu responsável, os objetivos da pesquisa e, para as que concordaram em participar, foi solicitada a assinatura do Termo de Consentimento Livre Esclarecido. Todas as adolescentes responderam a um questionário estruturado, composto por 65 perguntas diretas, com respostas fechadas, pré-codificadas, a serem assinaladas, investigando características sociodemográficas e socioeconômicas da adolescente e de seu companheiro, além da história obstétrica, dados de pré-natal, resultados perinatais e reações da adolescente, de sua família e do companheiro despertadas pela gestação. Um dos autores entregava à adolescente o questionário, dirigindo-se a ela exclusivamente quando solicitado a dirimir dúvidas. Os dados coletados foram organizados por meio do programa Epi-Info versão 6.04d, de janeiro de 2001, do Centers for Disease Control and Prevention ${ }^{12}$. Analisaram-se os dados empregando parâmetros de estatística descritiva e teste do $\chi^{2}$, em nivel de significância de 0,05, para análise inferencial.

A pesquisa foi autorizada pelo Comitê de Ética da Universidade de Pernambuco, em obediência à Resolução 196/96 do Conselho Nacional de Saúde ${ }^{14}$.

\section{Resultados}

Observamos que 527 (73,8\%) adolescentes tiveram uma única parturição, 149 (20,9\%) duas parturições, 35 (4,9\%) três partos e $3(0,4 \%)$ referiram quatro partos. Independente do número de partos, a via transvaginal predominou, com ou sem uso de fórcipe (Tabela 1). Dentre as primiparas e secundiparas houve 31 (5,9\%) e $4(2,7 \%)$ partos por fórcipe, respectivamente.

Tabela 1 - Distribuição das 714 puérperas segundo o número e a via de parto.

\section{Via de parto}

\begin{tabular}{crcrcrrr}
$\begin{array}{c}\text { Número de } \\
\text { partos }\end{array}$ & \multicolumn{2}{c}{ Transvaginal } & \multicolumn{2}{c}{ Cesárea } & \multicolumn{2}{c}{ Total } \\
& $\mathbf{n}$ & $\%$ & $\mathbf{n}$ & $\%$ & $\mathbf{n}$ & $\%$ \\
\hline 1 & 320 & 60,7 & 207 & 39,3 & 527 & 73,8 \\
2 & 95 & 63,7 & 54 & 36,3 & 149 & 20,9 \\
3 & 24 & 68,6 & 11 & 31,4 & 35 & 4,9 \\
4 & 2 & 66,7 & 1 & 33,3 & 3 & 0,4 \\
Total & 441 & 61,8 & 273 & 38,2 & 714 & 100,0 \\
\hline
\end{tabular}

Dentre as 273 gestantes submetidas a parto cesáreo, $207(75,8 \%)$ eram primiparas, 65 $(23,8 \%)$ paucíparas e $1(0,4 \%)$ multipara.

Comparando a via de parto de primeira parturição à da segunda, detectou-se concordância significante (Tabela 2). As parturientes estiveram expostas a risco aproximadamente 22 vezes maior de repetição da via do primeiro parto $(\mathrm{OR}=21,8 ; \mathrm{IC} 95 \%, 8,4-57,8)$.

Houve também associação estatisticamente significante entre as vias de parto de segunda e terceira parturições, tendo coincidido o parto transvaginal em $23(65,7 \%)$ adolescentes e cesárea 
em 10 (28,6\%) (Tabela 3). As gestantes com parto cesáreo na segunda parturição estiveram expostas a risco 230 vezes maior dessa via de parto na terceira parturição $(\mathrm{OR}=230$; IC 95\%, 10,1-10353,3).

Dado o pequeno número de multíparas, não foi possivel analisar a relação entre via do terceiro e do quarto partos, pois foram identificados dois partos transvaginais e uma cesárea em ambas as ocasiões.

Tabela 2 - Distribuição das 149 puérperas segundo a via de parto de primeira e segunda parturições.

\section{Via de parto da $2^{\mathrm{a}}$ parturição}

\begin{tabular}{lcccccc}
$\begin{array}{c}\text { Via de parto da } \\
\text { 1a } \text { parturição }\end{array}$ & \multicolumn{2}{c}{ Transvaginal } & \multicolumn{2}{c}{ Cesárea } & \multicolumn{2}{c}{ Total } \\
& $\mathbf{n}$ & $\%$ & $\mathbf{n}$ & $\%$ & $\mathbf{n}$ & $\%$ \\
\hline Transvaginal & 83 & 86,5 & 13 & 13,5 & 96 & 64,4 \\
Cesárea & 12 & 22,8 & 41 & 77,4 & 53 & 35,6 \\
Total & 95 & 63,7 & 54 & 36,3 & 149 & 100,0 \\
\hline
\end{tabular}

$\chi^{2}=57,45 \quad p<0,000$

$\mathrm{OR}=21,81$ IC $95 \%, 8,47-57,87$.

Tabela 3 - Distribuição das 35 puérperas segundo a via de parto de segunda e terceira parturições.

$$
\text { Via de parto da } 3^{\mathrm{a}} \text { parturição }
$$

\begin{tabular}{lrrrrrr}
$\begin{array}{c}\text { Via de parto da } \\
\mathbf{2}^{\mathbf{a}} \text { parturição }\end{array}$ & \multicolumn{2}{c}{ Transvaginal } & \multicolumn{2}{c}{ Cesárea } & \multicolumn{2}{c}{ Total } \\
& $\mathbf{n}$ & \multicolumn{1}{c}{$\%$} & $\mathbf{n}$ & $\%$ & $\mathbf{n}$ & $\%$ \\
\hline Transvaginal & 23 & 95,8 & 1 & 4,2 & 24 & 68,6 \\
Cesárea & 1 & 9,1 & 10 & 90,9 & 11 & 31,4 \\
Total & 24 & 68,6 & 11 & 31,4 & 35 & 100,0 \\
\hline
\end{tabular}

PFisher $<0,000$

OR = 230; IC 95\%; 10,13-10353,3

\section{Discussão}

A taxa de cesárea tem sido alvo de estudos nacionais e internacionais, que variam em virtude da metodologia adotada.

Yazlle et al. ${ }^{14}$, estudando número e tipo de parto de 7.134 adolescentes, atendidas no Município de Ribeirão Preto (SP), no período de janeiro de 1992 a dezembro de 1996, identificaram as porcentagens de partos transvaginais, fórcipe e cesáreas, respectivamente iguais a 59,2, 5,6 e $35,2 \%$. Atribuíram a freqüência de cesáreas a fatores que levaram à mudança da prática obstétrica no Brasil, quer como reflexo da elevação dessa taxa na população adulta, quer por expressivo número de intercorrências obstétricas.
Analisando 110 adolescentes, Kumbi e Isehak ${ }^{15}$ referiram taxa de cesárea de primiparidade de $21,8 \%$. Ribeiro et al. ${ }^{16}$, em estudo de 499 gestantes adolescentes, que pariram em 1994 em Ribeirão Preto, relataram taxa de cesárea de $35,9 \%$, assim como de parto instrumental igual a 7,0\%. Lao e $\mathrm{Ho}^{17}$, em estudo retrospectivo envolvendo 194 mães adolescentes, comparadas à população obstétrica atendida em 1997, identificaram que a incidência de cesárea nas pacientes adolescentes foi 4,1 vezes menor $(p<0,01)$. Galletta et al. ${ }^{18}$ referiram que o parto pélvico e o instrumental tiveram taxas aumentadas, em detrimento do parto normal, em 100 gestantes adolescentes, atendidas por equipe multidisciplinar no Hospital das Clínicas da Universidade de São Paulo.

Traçando o perfil epidemiológico das adolescentes (com idade média de 17 anos) que engravidaram na cidade de São Marcos (RS), Chemello $^{19}$ verificou percentual de cesárea de $55,6 \%$. Monteiro et al. ${ }^{20}$, em estudo tipo coorte para análise de modelo de assistência multidisciplinar à mãe adolescente, visando o bem-estar materno-fetal e a contracepção, independentemente da via de parto, observaram reincidência de gestação de $5,2 \%$, que consideraram baixa.

No presente estudo, considerando as 714 parturientes adolescentes (independentemente do número de gestações), as porcentagens igualaramse a $56,9,4,9$ e $38,2 \%$, respectivamente para parto transvaginal, instrumental e cesárea; foram pouco distintas daquelas de Yazlle et al. ${ }^{14}$, mas inferiores às de Silva e Nogueira ${ }^{1}$, os quais, analisando 157 pacientes com menos de 20 anos de idade, com uma ou mais gestações anteriores, no período entre janeiro de 1983 e dezembro de 1984, identificaram o uso três vezes superior de parto instrumental entre as primigestas $(32,5 \%)$.

Diferente de Silva e Nogueira ${ }^{1}$ e de Yazlle et al. ${ }^{14}$, não se fez a análise de cesárea iterativa nem se pode atribuir a taxa de cesárea a intercorrências obstétricas, pelo fato de as informações terem sido obtidas das pacientes, impossibilitando a consideração das causas que levaram à decisão médica dessa via de parto.

Silva e Nogueira ${ }^{1}$ não encontraram diferença significativa entre a via vaginal e a abdominal, mas referiram taxa de cesárea sensivelmente superior para as multigestas $(16,6 \%$ para primigestas contra $25,0 \%$ para multigestas), o que não se verificou no presente estudo.

O resultado mais interessante desta pesquisa parece ter sido a associação estatística entre as vias de parto de gestações subseqüentes, tendo havido tendência de se repetir a via de parto da gestação imediatamente antecedente, afirmação válida até a terceira gestação. 
Barbosa et al. ${ }^{21}$, em estudo sobre a existência de uma "cultura da cesárea" afirmaram que no Brasil houve um processo de legitimação social do parto cesáreo, tanto pelos médicos como pelas pacientes, como tipo de parto seguro, indolor, moderno e ideal para qualquer grávida. Mello e Souza ${ }^{23}$, em 1994, elucidou a forma pela qual valores culturais referentes à dor do parto vaginal, à imagem corporal feminina e ao fascínio pela tecnologia foram manipulados pela biomedicina a fim de acomodar essa prática dentro da orientação geral da medicina ocidental em relação à beneficência na qual o medo da dor passa a ser rotulado de "falta de preparo psicológico para o trabalho de parto", transformando-se em justificativa médica para a cesárea.

Mello e Souza ${ }^{23}$ argumentou que os médicos passaram a justificar a prática de cesáreas desnecessárias com base no princípio do direito da mulher ao poder e controle sobre o próprio corpo. Conforme essa interpretação, é o desejo feminino que determina o tipo de parto, e não o poder de promoção, legitimação e divulgação dos médicos.

Mesmo considerando essa abordagem fenomenológica, no presente estudo não se pode fazer tais afirmações, visto que não se investigaram as intercorrências obstétricas dessas adolescentes, para julgar a adequabilidade das indicações de cesárea. Mesmo assim, é preocupante a constatação de tal associação, ainda mais considerando-se a idade reprodutiva dessas parturientes.

Identificamos associação significante entre a via de parto entre a primeira e a segunda parturições, assim como entre a segunda e a terceira parturições. São necessários outros estudos para a identificação das possiveis causas dessa tendência dos serviços em supervalorizar a via do parto anterior.

\section{ABSTRACT}

Purpose: to analyze the association between modes of consecutive deliveries of 714 adolescents attended at the Maternity sector of the Hospital das Clínicas da Universidade Federal de Pernambuco (Federal University of Pernambuco), from January to December 2001.

Patients and Methods: according to a prospective, analytic, transversal, and incidence type study, the sequence of delivery modes was analyzed from the first to the fourth parturition of 714 pregnant adolescents, aged from 13 to 19 years (mean17.2 1.5 years). Every day, after identifying the adolescents who delivered, by the parturition room register, they were invited to participate in this study. Those who agreed, after signing a free informed consent answered a structured questionnaire with 65 direct questions with precodified closed options, among which were delivery mode and number of gestations.

Results: it was found that 527 (73.8\%) adolescents had only one delivery, 149 (20.9\%) two deliveries, 35 (4.9\%) three deliveries, and $3(0.4 \%)$ had had four deliveries. Among the 273 cesarean sections, 207 (75.8\%) occurred in primiparae, $65(23.8 \%)$ in secundiparae and one $(0.4 \%)$ in a multipara. There was a statistically significant association between the first and the second deliveries for 83 (55.7\%) adolescents who had transvaginal delivery, as well as for 41 (27.5\%) by cesarean section. There was also coincidence about the second and third deliveries, regarding transvaginal delivery of $23(65.7 \%)$ adolescents, as well as cesarean section of 10 (28.6\%) adolescents.

Conclusion: a tendency to coincidence of subsequent delivery modes was identified for adolescents until the third parturition.

KEYWORDS: Normal pregnancy. Adolescence. Cesarean section. Delivery.

\section{Referências}

1. Silva JLP, Nogueira CWM. A multigravidez na adolescência. In: OPS. Coletânea sobre Saúde Reprodutiva do Adolescente Brasileiro. Brasília: Organização Panamericana de Saúde; 1998. p. 101-11.

2. Wessel H, Cnattingius S, Bergstrom S, Dupret A, Reitmaier P. Maternal risk factors for preterm birth and low birthweight in Cape Verde. Acta Obstet Gynecol Scand 1996; 75:360-6.

3. Vitalle MSS. Adolescência e outros fatores de risco (nível econômico, cuidado pré-natal e tabagismo) como determinantes de prematuridade e baixo peso [tese]. São Paulo: Univ. Federal de São Paulo; 2001.

4. Gama SGN, Szwarcwald CL, Leal MC. Experiência de gravidez na adolescência, fatores associados e resultados perinatais entre puérperas de baixa renda. Cad Saúde Publica 2002; 18:153-61.

5. Gil MP. Aspecto médicos y sociales de la maternidad en la adolescencia. Rev Soc Chil Obstet Ginecol Infant Adolesc 2000; 7:16-25.

6. Azevedo GD, Freitas Júnior RAO, Freitas AKMSO, Araújo ACPF, Soares EMM, Maranhão TMO. O Efeito da idade materna sobre os resultados perinatais. Rev Bras Ginecol Obstet 2002; 24:181-5.

7. Alves AMPM. Partos em adolescentes: um perfil epidemiológico no município de João Pessoa PB, 1990-1999 [dissertação]. João Pessoa: Univ. Federal da Paraíba; 2001. 
8. Beretta MIR. Incidência de partos na adolescência na Cidade de São Carlos-SP. Rev Baiana Enfermagem 1998; 11:19-27.

9. Demir SC, Kadyýfcý O, Özgünen T, et al. Pregnancy outcomes in young Turkish women. J Pediatr Adolesc Gynecol 2000; 13:177-81.

10. Costa MCO, Santos CAT, Sobrinho CL, Freitas JO, Ferreira KASL. Indicadores materno-infantis na adolescência e juventude: sociodemográfico, prénatal, parto e nascidos-vivos. J Pediatr (RJ) 2001; 77:235-42.

11.Torres Ramírez A, Coria Soto IL, Zambrana MM, Lara Ricalde $R$. La resolución obstétrica de las adolescentes en comparación con la de las adultas. Ginecol Obstet Mex 1999; 67:377-84.

12.WHO. Epi Info [computer program]. Version 6.04d. Geneva: Centers for Disease Control and Prevention; 2001.

13. Ministério da Saúde. Resolução nº 196, out. 1996. Brasília: DOU; 1996.

14.Yazlle MEHD, Mendes, MC, Patta MC, Rocha JSY, Azevedo GD, Marcolin AC. A adolescente grávida: alguns indicadores sociais. Rev Bras Ginecol Obstet 2002; 24:609-14.
15.Kumbi S, Isehak A. Obstetric outcome of teenage pregnancy in northwestern Ethiopia. East Afr Med J 1999; 76:138-40.

16. Ribeiro ERO, Barbieri MA, Bettiol H, Silva AAM. Comparação entre duas coortes de mães adolescentes em município do Sudeste do Brasil. Rev Saúde Pública 2000; 34:136-42.

17.Lao TT, HO LF. Obstetric outcome of teenage pregnancies. Hum Reprod 1998; 13:3228-32.

18. Galleta MAK, Lippi ATA, Giribola A, Mígueles J, Zugaib M. Resultados obstétricos e perinatais em gestantes adolescentes atendidas em pré-natal especializado. Rev Ginecol Obstet 1997; 8:10-9.

19. Chemello CS. Perfil epidemiólogico das adolescentes grávidas na cidade de São Marcos, RS [dissertação]. São Paulo: Univ. de São Paulo; 1999.

20. Monteiro DLM, Cunha AA, Fagim IG, Paiva AS. Modelo de assistência multidisciplinar na adolescência, visando o bem estar materno-fetal e a contracepção. Reprod Clim 1995; 10:67-72.

21.Barbosa GP, Giffin K, Angulo-Tuesta A, et al. Parto cesáreo: quem o deseja? Em quais circunstâncias? Cad Saúde Pública 2003; 19:1611-20.

Recebido em: 10/10/03 Aceito com modificações em: 21/10/04 